\title{
Pruebas ecotoxicológicas con aguas de lastre y gestión de los residuos marinos procedente de buques
}

\author{
Ecotoxicological tests with ballast waters and marine waste management \\ from ships
}

\author{
Alfonso Ramírez Caján 1; Verónica Espinel Pino ${ }^{2}$
}

Recibido: Junio 2019 - Aprobado: Julio 2019

\begin{abstract}
RESUMEN
Esta investigación presenta resultados de los efectos ecotoxicológicos de las aguas de lastre transportada por los buques que arriban de viajes internacionales al puerto del Callao sobre juveniles de Odontesthes regia regia (Pejerrey), mediante un bioensayo agudo que determinó la mortandad de más del $50 \%$ de los organismos a partir de la concentración $\mathrm{C}_{3}$; asi como de una evaluación de parámetros fisicoquímicos y microbiológicos que reveló valores de $\mathrm{pH}$ fuera del rango normal de las aguas marinas con presencia de bacterias heterótrofas en cantidades de 210 unidades. Asimismo, se evaluó la gestión de los residuos que los buques dispusieron a través de artefactos navales e instalaciones de recepción acuática durante los años del 2017 al 2019 en 11 puertos marítimos del Perú, siendo el puerto del Callao el mayor receptor con 1,044.95 m³ de mezclas oleosas y 385.71 $\mathrm{t}$ de residuos y el puerto de Chancay el de menor transferencia con $28.99 \mathrm{~m}^{3}$ de mezclas oleosas y $16.19 \mathrm{t}$ de residuos, debido a la presencia de un mayor número de buques en la flota naviera y a la concentración de actividades acuáticas en cada zona de operación.
\end{abstract}

Palabras clave: Agua de lastre; artefactos navales; basura marina; bioensayo; buque; efectos ecotoxicológicos.

\section{ABSTRACT}

This research focused on the analysis of the ecotoxicological effects of ballast water transported by ships arriving from international trips to the port of Callao on juveniles of Odontesthes regia regia (Pejerrey), through an acute bioassay that determined the mortality of more than $50 \%$ of the organisms from the $\mathrm{C}_{3}$ concentration; as well as an evaluation of physicochemical and microbiological parameters that revealed pH values outside the normal range of marine waters with the presence of heterotrophic bacteria in quantities of 210 units. Likewise, the management of the waste that the ships disposed of through naval devices and aquatic reception facilities during the years of 2017 to 2019 in 11 seaports of Peru was evaluated, being the port of Callao the largest receiver with $1,044.95 \mathrm{~m} 3$ of mixtures oily and $385.71 \mathrm{t}$ of waste and the port of Chancay the one of lower transfer with 28.99 $\mathrm{m} 3$ of oily mixtures and $16.19 \mathrm{t}$ of waste, due to the presence of a greater number of ships in the shipping fleet and to the concentration of aquatic activities in each zone of operation.

Keywords: Ballast water; naval artifacts; marine garbage; bioassay; ship; ecotoxicological effects.

\footnotetext{
${ }_{1}$ Universidad Alas Peruanas. Lima, Perú. Ingeniero Ambiental. Lima, Perú. E-mail: alfonso.ramirezcajan@unmsm.edu.pe

2 Universidad Técnica de Manabí. Manabí, Ecuador. Ingeniera Ambiental. E-mail: verie27@hotmail.es 


\section{INTRODUCCIÓN}

Las mareas rojas constituyen fenómenos naturales y antrópicos que se han manifestado a lo largo de la historia, causando impactos negativos a las pesquerías, ecosistemas y salud humana. (Torres, 2015). Los ensayos biológicos son herramientas de diagnóstico adecuadas para determinar el efecto de agentes físicos y químicos sobre organismos de prueba bajo condiciones experimentales específicas y controladas. (Castillo, 2004). La variabilidad genética del pejerrey Odontesthes regia es elevada y presenta un gradiente de disminución de sur a norte. (Deville, 2016). El tiempo que demora en eclosionar las ovas de pejerrey está supeditado a la variación de temperatura, demorando en su ambiente natural entre 35 a 45 días, mientras que en ambientes controlados 9 días con simulaciones de $17,5^{\circ} \mathrm{C}$ a $22,3{ }^{\circ} \mathrm{C}$. (Ganoza et al., 2014)

El transporte acuático permite la introducción de especies exóticas invasoras a nuevos entornos marinos. Las aguas de lastre procedente de los buques, tienen por función mantener la estabilidad del peso y asiento de los mismos. (Organización Marítima Internacional, 2005). Las descargas no controladas al mar, ocasiona la transferencia de organismos acuáticos perjudiciales que suponen una amenaza en la cadena trófica, por lo cual es necesario adoptar medidas seguras y eficaces para su gestión, a fin de reducir al mínimo y eliminar el intercambio de agentes patógenos. (Ramírez, 2015)

Las instalaciones de recepción acuática como las situadas a bordo de un buque o artefacto naval, son una importante infraestructura para el almacenamiento temporal de los residuos y mezclas oleosas, a fin de prevenir la contaminación acuática. Los residuos acopiados de manera segregada son dispuestos en instalaciones portuarias mediante embarcaciones de servicio; mientras que los residuos líquidos son evacuados a través de tuberías diseñadas para recibir los lastres sucios y/o slops, para su posterior tratamiento. (Salcedo, 2016)

Los principales puertos que albergan la mayor flota de embarcaciones en el Perú son el Callao, Chimbote, Paita e Ilo; sin embargo el deficiente número de instalaciones dedicadas a la recepción de los residuos operacionales de los buques, no permite llevar una adecuada gestión de protección marina y de sus recursos naturales, más aún en aquellos puertos fluviales y lacustres donde no existen estos mecanismos de facilidades de recepción acuática.

\section{MÉTODOS}

\subsection{Diseño del estudio}

2.1.1 Pruebas ecotoxicológicas de aguas de lastre

Para determinar los efectos ecotoxicológicos de las aguas de lastre sobre la Odontesthes regia regia (tasa de mortandad), se llevó a cabo una investigación experimental. El diseño del estudio utilizó un grupo control y un grupo experimental sometidos a cinco concentraciones de agua de lastre de un buque. (Instituto del Mar del Perú, 2017).

Para la aleatorización de las muestras a diferentes concentraciones se utilizó el diseño de bloque:
C: Concentración

$0,1,2,3,4,5$ : Tratamiento de menor a mayor

A, B, C, D: Réplica de la muestra

\begin{tabular}{|l|l|l|l|}
\hline $\mathrm{C} 1 \mathrm{~A}$ & $\mathrm{C} 3 \mathrm{~A}$ & $\mathrm{C} 4 \mathrm{C}$ & $\mathrm{C} 2 \mathrm{C}$ \\
\hline $\mathrm{C} 4 \mathrm{~A}$ & $\mathrm{C} 2 \mathrm{~A}$ & $\mathrm{C} 1 \mathrm{~B}$ & $\mathrm{C} 5 \mathrm{C}$ \\
\hline $\mathrm{C} 3 \mathrm{C}$ & $\mathrm{C} 5 \mathrm{D}$ & $\mathrm{C} 4 \mathrm{D}$ & $\mathrm{C} 3 \mathrm{~B}$ \\
\hline $\mathrm{C} 5 \mathrm{~B}$ & $\mathrm{C} 0 \mathrm{~A}$ & $\mathrm{C} 2 \mathrm{~B}$ & $\mathrm{C} 3 \mathrm{D}$ \\
\hline $\mathrm{COD}$ & $\mathrm{C} 4 \mathrm{~B}$ & $\mathrm{C} 1 \mathrm{C}$ & $\mathrm{C} 5 \mathrm{~A}$ \\
\hline $\mathrm{COB}$ & $\mathrm{C} 1 \mathrm{D}$ & $\mathrm{C} 2 \mathrm{D}$ & $\mathrm{C} C \mathrm{C}$ \\
\hline
\end{tabular}

\subsubsection{Gestión de residuos marinos}

La investigación fue no experimental con método transversal, porque se recolectaron datos de la disposición de los residuos de los buques en un solo momento durante los años del 2017 al 2019, a fin de analizar su incidencia e interrelación con la realidad portuaria.

\subsection{Instrumentos empleados}

Para las pruebas ecotoxicológicas se utilizó una balanza analítica marca Dakota y un ictiómetro Krauss \& Henke, a fin de determinar el peso y la talla de los organismos; así como un multiparámetro marca Analytics y un conductímetro marca Inolab para la medición de los parámetros fisicoquímicos; además de frascos colectores de $120 \mathrm{ml}$ para la obtención de las muestras y 2 acuarios de 1201 para el acondicionamiento y eclosión de las ovas (Figura 1).

Para la obtención de datos de los volúmenes de descargas y pesos de los residuos recibidos por las instalaciones de recepción acuática, se emplearon los reportes trimestrales que los propietarios de dichas instalaciones remiten a las Capitanías de Puerto de la Dirección General de Capitanías y Guardacostas; así como la copia de los certificados de matrícula de los artefactos navales (chatas) que dicha autoridad otorga. (Madariaga, 2010) (Figura 2)

\subsection{Procedimientos de recolección de datos}

\subsubsection{Datos para pruebas ecotoxicológicas}

La colecta de ovas de Odontesthes regia regia se realizó en inmediaciones de la playa Isla Galápagos, cuyo material biológico se encontraba adherido a la Ulva lactuca (alga verde), siendo posteriormente transportadas al Laboratorio de Ecotoxicología Acuática del Instituto del Mar del Perú para su acondicionamiento hasta su eclosión. Las muestras de aguas de lastre fueron tomadas del buque petrolero quimiquero "Chira", identificado con número de matrícula CO-40533-MM, ubicado en el área "8" de la zona de fondeo para buques tanques (Figura 3).

La medición de los parámetros fisicoquímicos de oxígeno disuelto, $\mathrm{pH}$, temperatura, conductividad eléctrica y salinidad fueron efectuados in situ., mientras que para el muestreo a nivel microbiológico se extrajeron muestras en 


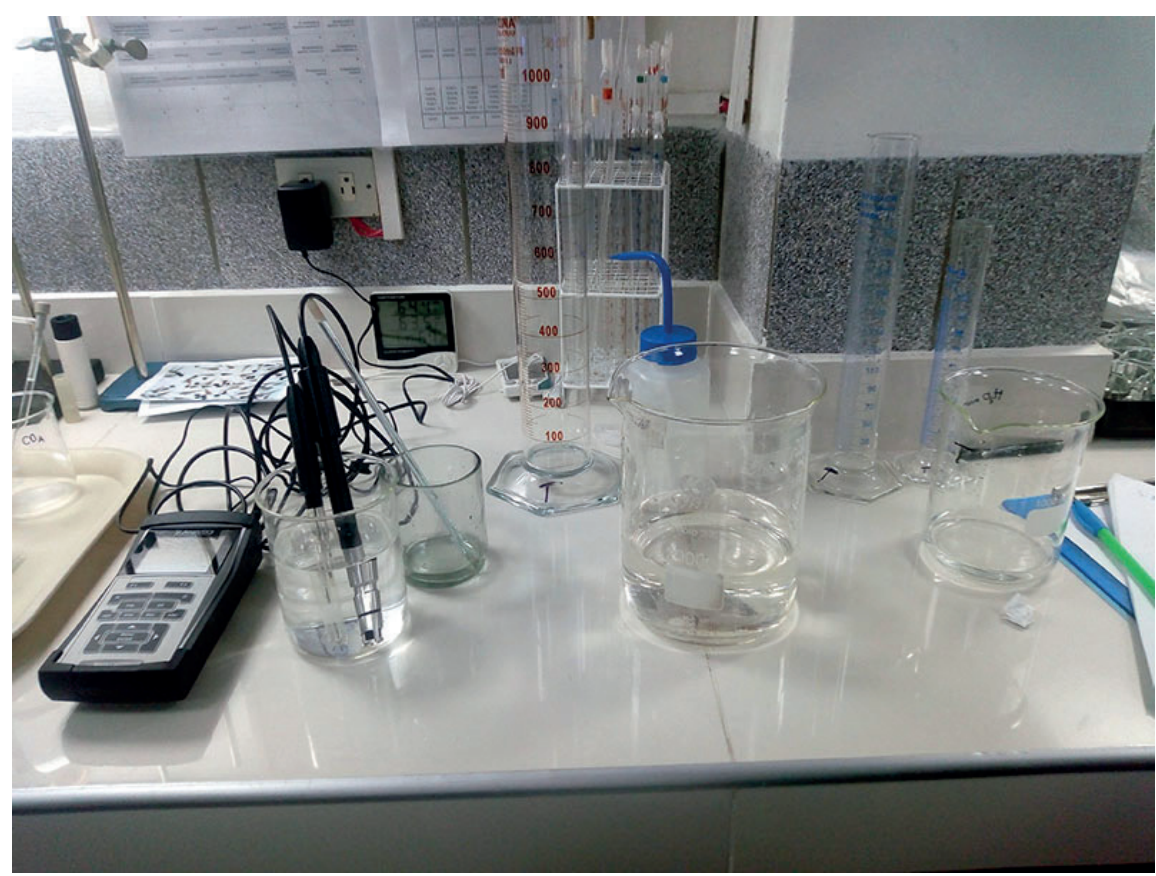

Figura 1. Materiales e instrumentos de medición y análisis

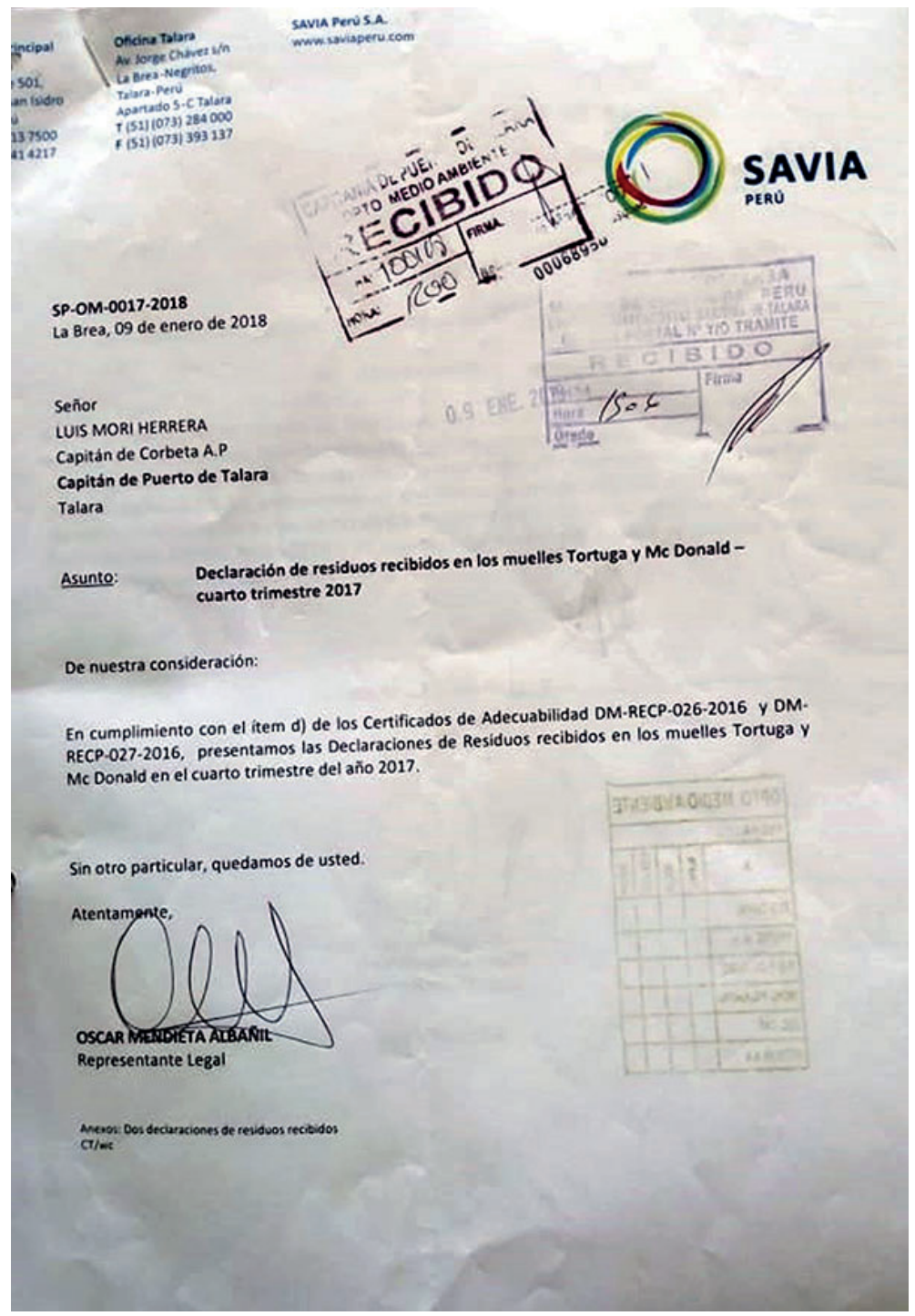

Figura 2. Reporte trimestral de residuos 


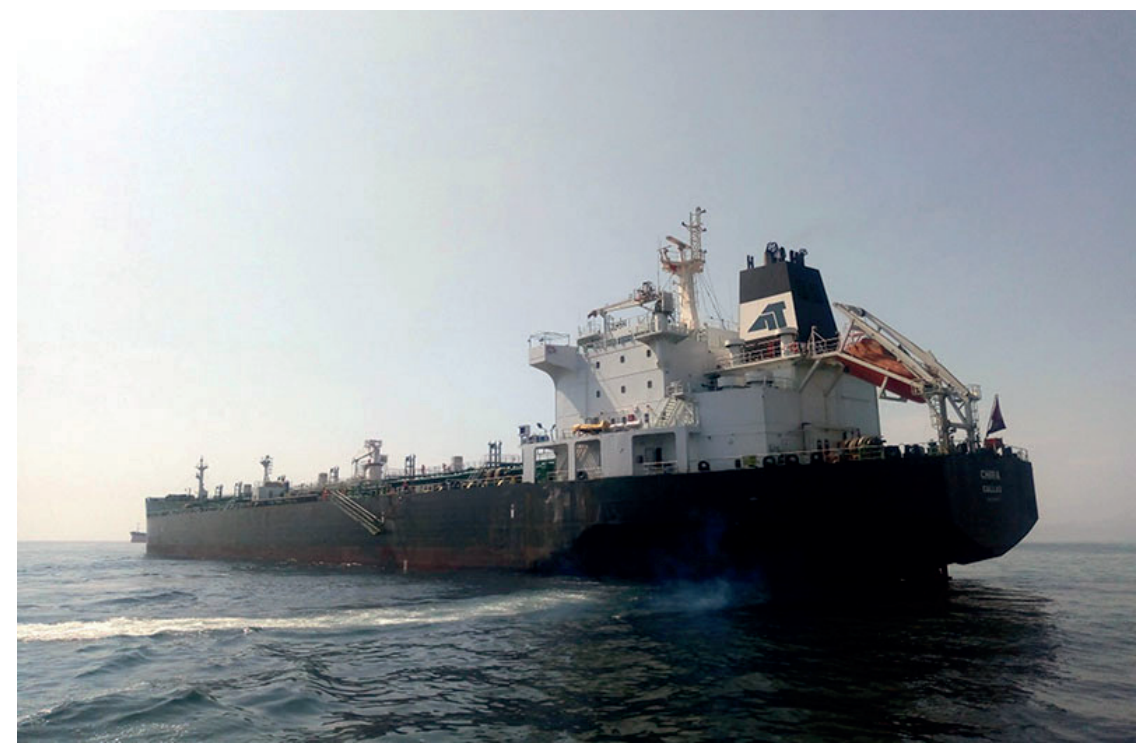

Figura 3. Estación de muestreo - Buque mercante "Chira"

vasos de precipitado de $300 \mathrm{ml}$, para su posterior análisis en laboratorio y determinación del número de Escherichia coli, Enterococos intestinales, Vibrio cholerae y bacterias heterótrofas. (Sánchez, G. y Vera, G.2001) (Figura 4).

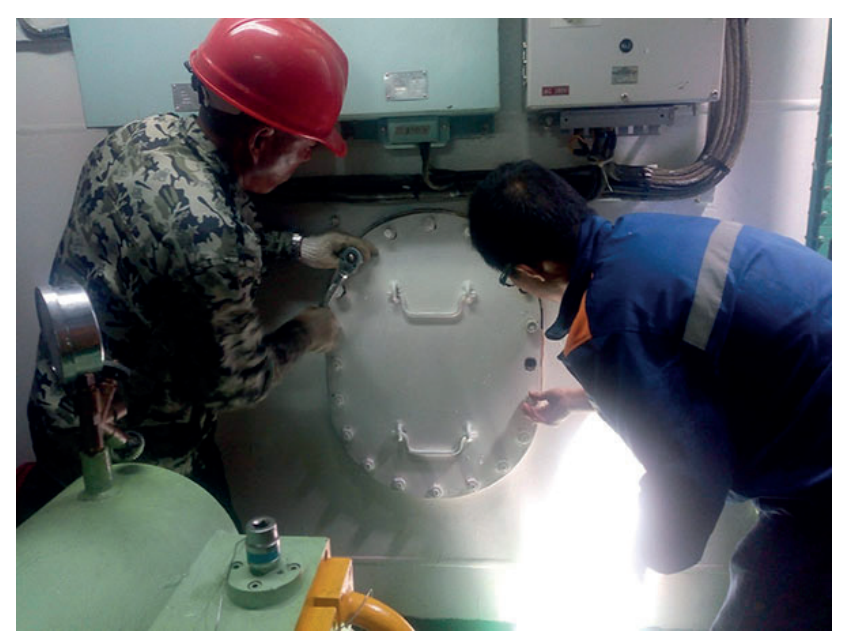

Figura 4. Punto de muestreo de agua de lastre

En las pruebas ecotoxicológicas con aguas de lastre (Figura 5), se utilizaron postlarvas de Odontesthes regia regia (Pejerrey) de 30 días de eclosionadas de estadío juvenil de $0,142 \mathrm{~g} \pm 0,04$ de peso y $25,10 \mathrm{~mm} \pm 1.5$ de longitud, las cuales fueron colocadas en cantidades de 10 individuos por unidad de estudio en cada una de las 4 réplicas, acorde a los criterios de aceptabilidad para pruebas de toxicidad de la Tabla 1. Durante el bioensayo los organismos no se alimentaron, se contó el número de sobrevivientes al cabo de 24, 48, 72 y 96 horas, según la prueba multiconcentración de ensayo agudo (mortandad) de la Tabla 2 (Orosco, P. y Villanueva, H. 2015). Se consideró muertos a aquellos que no tenían movimiento y se encontraban asentados en el fondo del vaso de precipitado, de acuerdo a la metodología USEPA (Instituto del Mar del Perú, 2017)

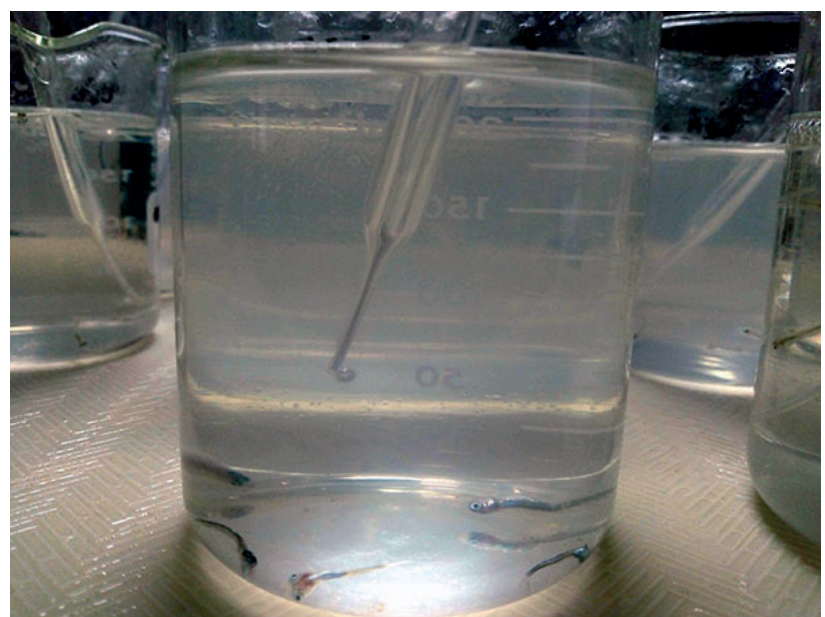

Figura 5. Efectos de prueba ecotoxicológica

Tabla 1. Parámetros fisicoquímicos de agua de lastre

\begin{tabular}{ccccc}
\hline $\begin{array}{c}\mathrm{OD} \\
(\mathrm{mg} / \mathrm{l})\end{array}$ & $\mathrm{pH}$ & $\begin{array}{c}\text { Temp. } \\
\left({ }^{\circ} \mathrm{C}\right)\end{array}$ & $\begin{array}{c}\text { Cond. Eléct. } \\
(\boldsymbol{\mu S} / \mathrm{cm})\end{array}$ & $\begin{array}{c}\text { Salinidad } \\
(\mathrm{UPS})\end{array}$ \\
\hline 7.93 & 10.48 & 22.9 & 376 & 0.1 \\
\hline
\end{tabular}

Tabla 2. Parámetros microbiológicos de agua de lastre

\begin{tabular}{cccc}
\hline $\begin{array}{c}\text { E. coli (UFCl } \\
100 \mathrm{~mL})\end{array}$ & $\begin{array}{c}\text { Enterococos } \\
\text { intestinales } \\
(\mathrm{P} \circ \mathrm{A} / 100 \mathrm{~mL})\end{array}$ & $\begin{array}{c}\text { Vibrio cholerae } \\
\text { (P ० A } / 100 \mathrm{~mL})\end{array}$ & $\begin{array}{c}\text { Bacterias } \\
\text { heterótrofas } \\
\text { (UFC } / 100 \mathrm{~mL})\end{array}$ \\
\hline$<1$ & Ausencia & Ausencia & 210 \\
\hline
\end{tabular}

2.3.1 Datos para gestión de residuos de buques

Se accedió a la información a través de las 11 Capitanías de Puerto pertenecientes a la Dirección General de Capitanías y Guardacostas, cuyos reportes trimestrales de disposición de residuos entregados por los propietarios de los artefactos navales e instalaciones acuáticas, se encuentran establecidos mediante Resolución Directoral No 766-2003/DCG de fecha 31 de diciembre del 2003, sobre las facilidades de recepción de residuos generados por los buques (Figura 6 y 7 ). 


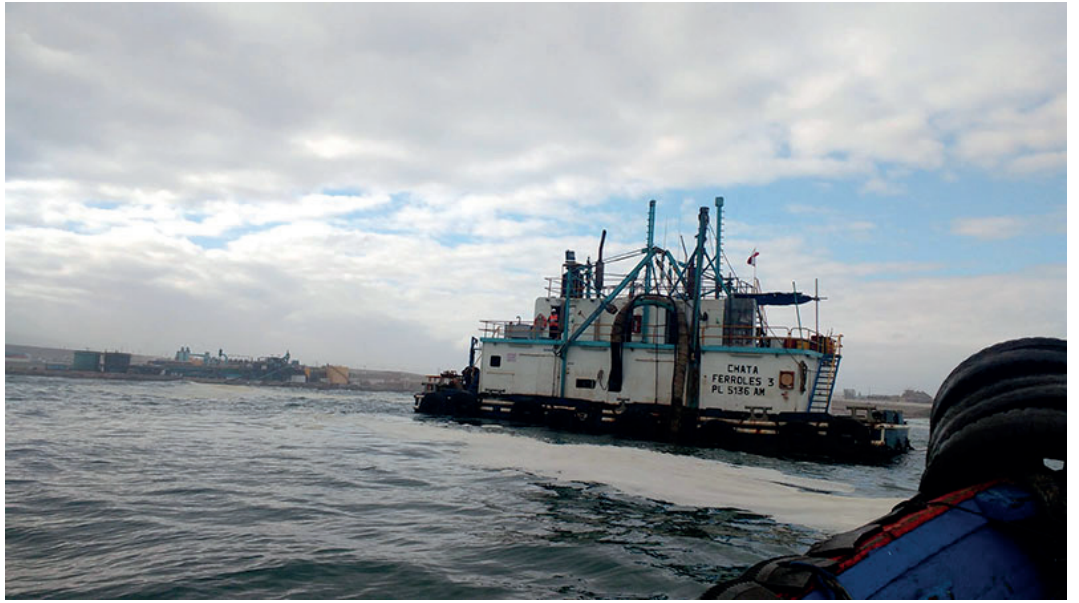

Figura 6. Artefacto Naval "Chata Ferroles 3"

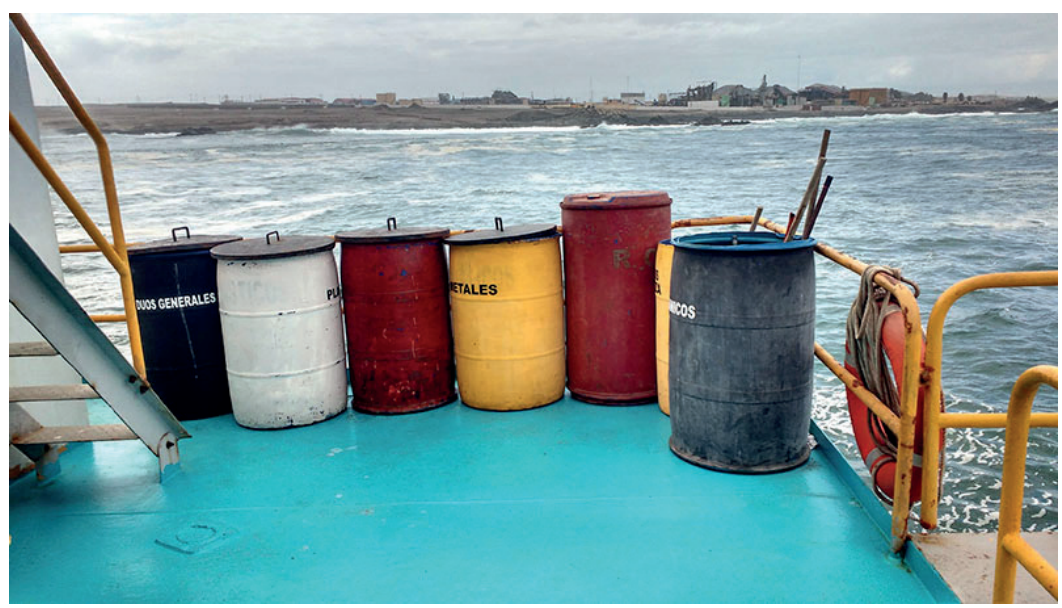

Figura 7. Recepción de residuos - Ilo

\section{RESULTADOS}

\subsection{Pruebas ecotoxicológicas}

Durante el bioensayo la mortandad de los individuos se registró en función al grado de concentración, obteniéndose para $\mathrm{C}_{0} 0, \mathrm{C}_{1} 2, \mathrm{C}_{2} 3, \mathrm{C}_{3} 5, \mathrm{C}_{4} 8$ y $\mathrm{C}_{5} 10$, encontrándose la concentración letal media $\left(\mathrm{CL}_{50}\right)$ en la concentración $\mathrm{C}_{3}$. Los individuos sobrevivientes presentaron síntomas de letargo y nado anormal, con movimiento brusco y rápido de sus aletas y rozamiento de sus cuerpos con el recipiente que los contenía.

\subsection{Gestión de residuos de buques}

\subsubsection{Gestión de las mezclas oleosas}

Los reportes trimestrales de disposición de residuos oleosos fueron comparados con los recibos de recepción entregados por los propietarios de las instalaciones acuáticas y artefactos navales (chatas), mediante los cuales se consigna el lugar y hora de la recepción, el volúmen de descarga en metros cúbicos y el nombre y número de matrícula del buque que dispone el residuo, quedando además asentada dicha información en el Libro de Registro de Hidrocarburos y/o Diario de Navegación, que los buques mantienen a bordo tras cada operación como mínimo un año, para efectos de verificación por parte de la Autoridad
Marítima Nacional. (Organización Marítima Internacional, 1973). Las descargas de los residuos oleosos realizados desde los artefactos navales a tierra se llevaron a cabo mediante tuberías subacuáticas (Figura 8).

La tendencia en el incremento de las descargas de mezclas oleosas dispuestas por los buques durante los años del 2017 al 2018 fue creciente para los puertos de Talara, Supe, Chancay y Callao, mientras que esta tendencia se manifestó en sentido inverso para los puertos de Paita, Salaverry, Chimbote, Huacho, Pisco, Mollendo e Ilo, debido al movimiento transitorio que realizan los buques anualmente en temporadas de pesca, transporte de mercancías y otras actividades en el mar (Figura 9, Tabla 3).

Debido a que el puerto del Callao concentra la mayor parte de la flota naviera en el Perú, éste presentó mayor demanda de recepción y disposición de mezclas oleosas generadas por los buques durante los años del 2017 al 2019 con $1,044.95 \mathrm{~m}^{3}$, seguido del puerto de Chimbote con 293.03 $\mathrm{m}^{3}$, Ilo con $206.60 \mathrm{~m}^{3}$, Pisco con $200.14 \mathrm{~m}^{3}$, Mollendo con $185.87 \mathrm{~m}^{3}$, Talara con $159.52 \mathrm{~m}^{3}$, Huacho con $108.10 \mathrm{~m}^{3}$, Paita con $97.12 \mathrm{~m}^{3}$, Supe con $89.06 \mathrm{~m}^{3}$, Salaverry con 84.05 $\mathrm{m}^{3}$ y Chancay con $28.99 \mathrm{~m}^{3}$ (Figura 10). 


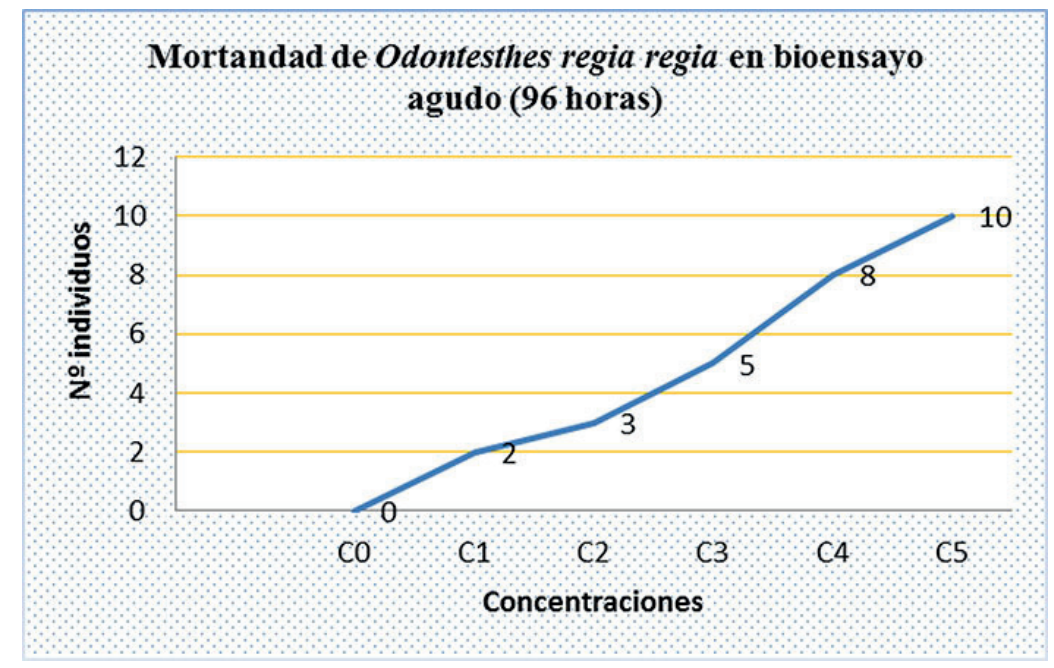

Figura 8. Mortandad de Odontesthes regia regia

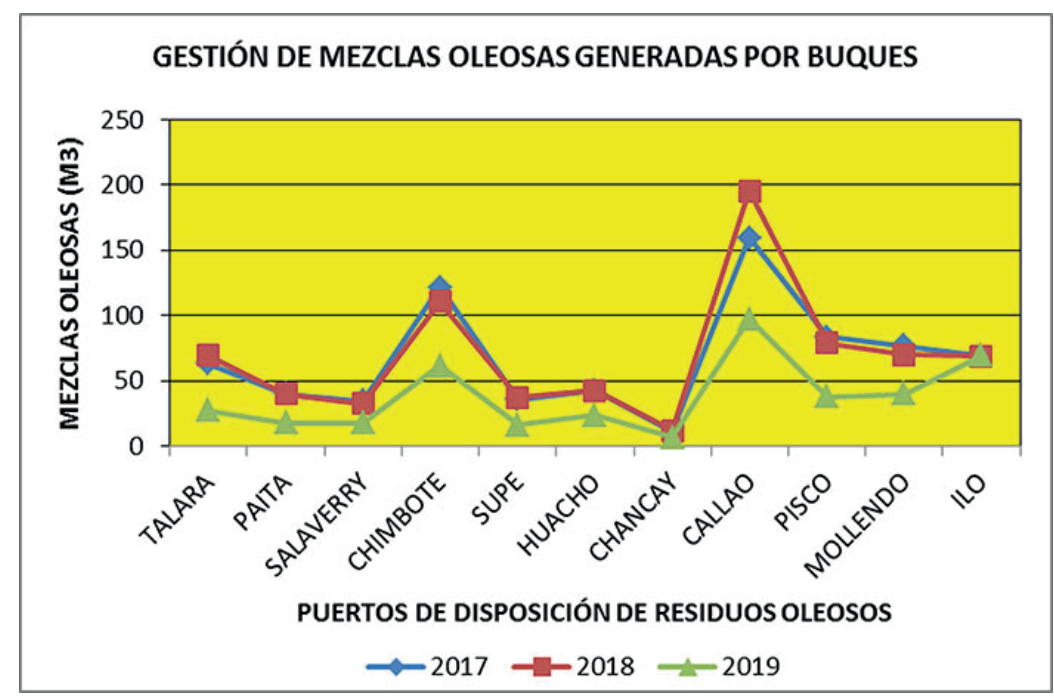

Figura 9. Gestión de mezclas oleosas de buques

Tabla 3. Registro de recepción de mezclas oleosas

\begin{tabular}{lccc}
\hline Puerto & \multicolumn{3}{c}{ Mezclas oleosas $\left.\mathbf{( m}^{3}\right)$} \\
\hline Talara & $\mathbf{2 0 1 7}$ & $\mathbf{2 0 1 8}$ & $\mathbf{2 0 1 9}$ \\
Paita & 63.15 & 69.52 & 26.85 \\
Salaverry & 39.95 & 39.62 & 17.55 \\
Chimbote & 34.22 & 32.37 & 17.46 \\
Supe & 121.37 & 110.39 & 61.27 \\
Huacho & 35.73 & 37.26 & 16.07 \\
Chancay & 42.46 & 42.18 & 23.46 \\
Callao & 10.98 & 11.74 & 6.27 \\
Pisco & 159.68 & 195.45 & 97.21 \\
Mollendo & 83.51 & 78.84 & 37.79 \\
Ilo & 76.42 & 69.69 & 39.76 \\
\hline
\end{tabular}




\section{CANTIDAD DE RESIDUOS OLEOSOS GENERADOS POR PUERTO}
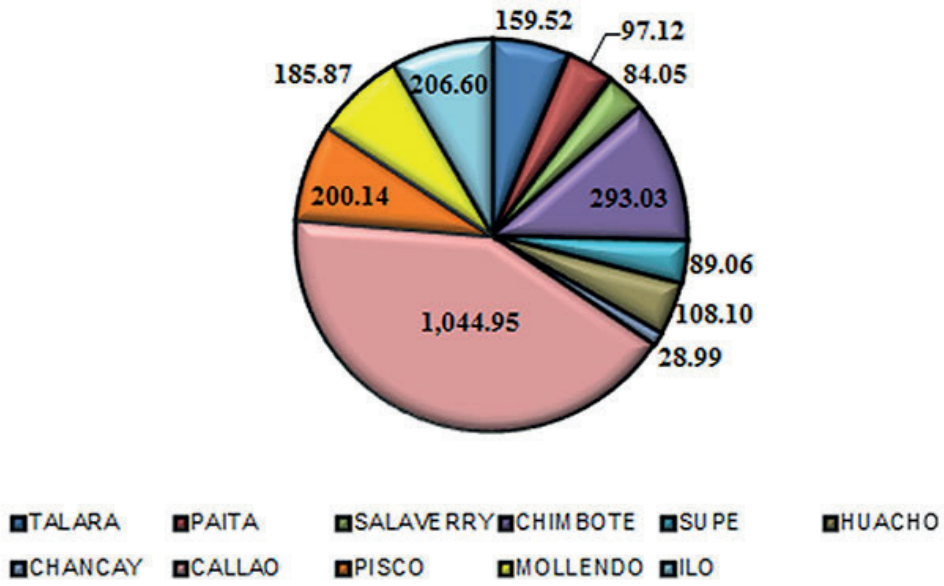

Figura 10. Residuos oleosos dispuestos en los puertos del Perú

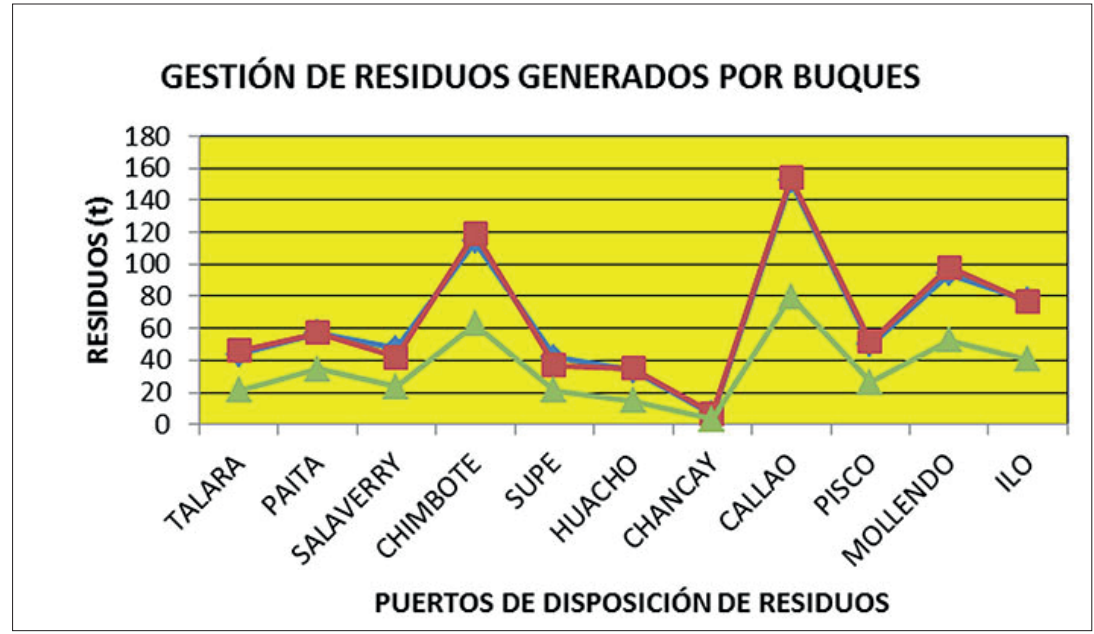

Figura 11. Gestión de residuos sólidos de buques

\subsubsection{Gestión de residuos}

Lo residuos generados a bordo de los buques fueron segregados según su naturaleza orgánica e inorgánica y depositados en recipientes rígidos a bordo de artefactos navales (chatas), mediante los cuales se dispuso los residuos en bolsas plásticas de colores selladas y transportadas hacia instalaciones acuáticas ubicadas en tierra para su tratamiento (Figura 11).

La tendencia en el incremento de recolección de residuos dispuestos por los buques durante los años del 2017 al 2018 es creciente para los puertos de Talara, Chimbote, Huacho, Chancay, Callao, Pisco y Mollendo; mientras que esta tendencia se manifiesta en sentido inverso para los puertos de Paita, Salaverry, Supe e Ilo, debido al movimiento transitorio que realizan los buques anualmente en temporadas de pesca, transporte de mercancías y otras actividades en el mar (Tabla 4).
Tabla 4. Registro de recepción de residuos sólidos

\begin{tabular}{lccc}
\hline Puerto & \multicolumn{3}{c}{ Residuos generados (t) } \\
& $\mathbf{2 0 1 7}$ & $\mathbf{2 0 1 8}$ & $\mathbf{2 0 1 9}$ \\
\hline Talara & 43.85 & 46.08 & 21.04 \\
Paita & 56.93 & 56.92 & 34.53 \\
Salaverry & 47.22 & 41.8 & 23.33 \\
Chimbote & 114.07 & 119.41 & 62.73 \\
Supe & 42.02 & 36.63 & 21.38 \\
Huacho & 33.5 & 35.17 & 14.76 \\
Chancay & 6.34 & 6.84 & 3.01 \\
Callao & 152.23 & 153.95 & 79.53 \\
Pisco & 49.63 & 51.77 & 26.24 \\
Mollendo & 94.13 & 98.05 & 52.32 \\
Ilo & 77.57 & 76.54 & 40.56 \\
\hline
\end{tabular}


La disposición de los residuos considerados como restos de víveres y residuos de faenas domésticas y trabajos rutinarios del buque en condiciones normales de servicio, durante los años del 2017 al 2019 representó en el puerto del Callao un $21 \%$ (385.71 t) del total de los residuos que se generaron en los 11 puertos marítimos identificados, seguido de Chimbote con un 16\% (296.22 t), Mollendo con un $13 \%(244.51 \mathrm{t})$, Ilo con un 11\% (194.68 t), Paita con un $8 \%$ (148.39 t), Pisco con un 7\% (127.65 t), Talara (110.98 t), Salaverry $(112.37 \mathrm{t})$ y Supe con un $6 \%$ (100.04 t), Huacho con un $5 \%(83.44 \mathrm{t})$ y Chancay con un $1 \%$ (16.19 t) (Figura 12, Tabla 5).

\section{DISCUSIÓN}

De acuerdo a bioensayos realizados con diferentes especies de peces, se determinó que la Odontesthes regia regia es más sensible. (Vera, Tam y Pinto 2001). Durante la prueba de toxicidad aguda se observaron abundantes excretas, además de problemas respiratorios y movimientos operculares rápidos en dichos organismos. Previo al bioensayo los resultados de los parámetros físico químicos fueron de $16{ }^{\circ} \mathrm{C}$ de temperatura, $6 \mathrm{mg} / 1$ de oxígeno disuelto, 8.11 de $\mathrm{pH}$ y 35 UPS de salinidad, determinándose que a mayor concentración del tóxico existe mayor mortandad. Si bien los parámetros fisicoquímicos y microbiológicos de las aguas de lastre presentan diferencias específicas respecto al agua del mar, la presencia de especies foráneas a nivel de fitoplancton y zooplancton representan un riesgo significativo para la biota acuática (Tabla 6).

Por otro lado, la gestión de los residuos de los buques en el Perú indica un número importante de residuos operacionales generados a bordo; sin embargo, existe un importante déficit de artefactos navales e instalaciones acuáticas de recepción de residuos en comparación al número de embarcaciones de bandera nacional y extranjeras que operan en nuestro territorio marítimo, además de la presencia de otros tipos de residuales como los lodos y sedimentos marinos ubicados en los tanques de la sala de máquinas de dichas naves, que no son dispuestos ni tratados, creando incertidumbre en la ruta de la basura acuática. (Marchant, 2009) (Tabla 7)

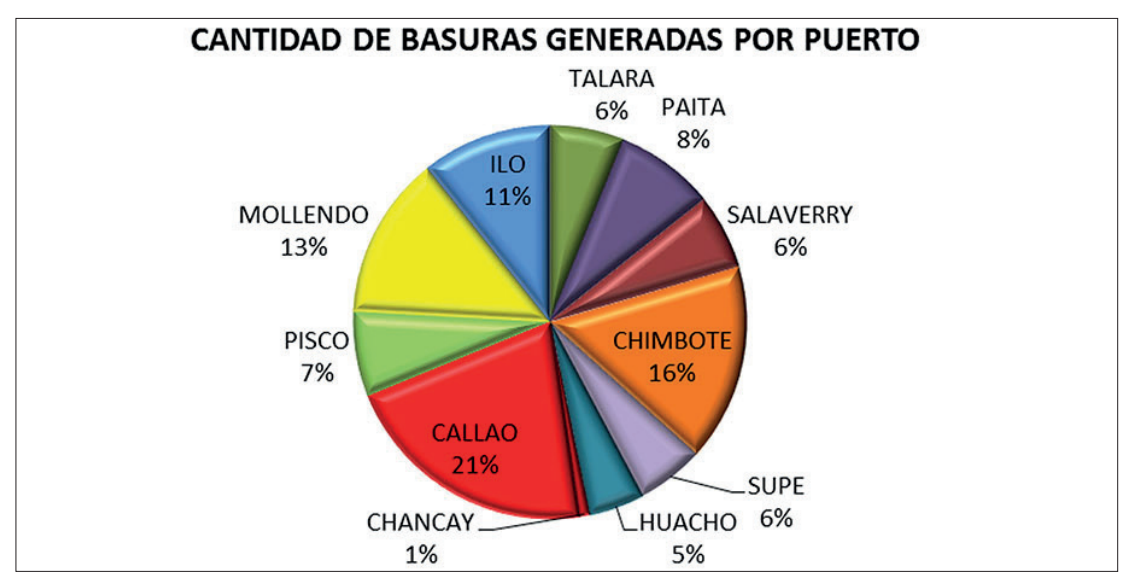

Figura 12. Basuras dispuestas en los puertos del Perú

Tabla 5. Criterios de aceptabilidad para prueba de toxicidad

\begin{tabular}{ll}
\hline Organismo de prueba & Odontesthes regia regia (Pejerrey) \\
Tipo de prueba & Estática sin recambio, 96 horas \\
Aireación & No requiere solo si ésta baja de $6 \mathrm{mg} / \mathrm{l}$ \\
Agua de dilución & Agua de mar filtrada \\
Salinidad & $35 \%$ \\
Temperatura & $20 \pm 2^{\circ} \mathrm{C}$ \\
Fotoperiodo & 12 horas luz : 12 horas oscuridad \\
Edad de los organismos de prueba & Estadío juvenil \\
Número de organismos por muestra & 10 individuos \\
Número de réplicas por concentración & 4 réplicas \\
Alimentación & No \\
Concentraciones de prueba & 5 \\
Respuesta & Mortandad \\
Criterio de aceptabilidad de prueba & $90 \%$ o más sobrevivientes en el control \\
\hline
\end{tabular}


Tabla 6. Prueba multiconcentración de ensayo agudo (mortandad)

\begin{tabular}{|c|c|c|c|c|c|c|c|}
\hline \multirow{3}{*}{$\begin{array}{l}\text { 䒕 } \\
\text { ठัँ } \\
\text { ठั }\end{array}$} & \multirow{3}{*}{ ळ } & \multirow{3}{*}{$\frac{\mathscr{d}}{\frac{\mathbb{0}}{0}}$} & \multicolumn{4}{|c|}{ Fechas / Horas } & \multirow{3}{*}{ 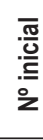 } \\
\hline & & & 24 hrs. & 48 hrs. & 72 hrs. & 96 hrs. & \\
\hline & & & 16-Abr & 17-Abr & 18-Abr & 19-Abr & \\
\hline \multirow{4}{*}{ CO } & \multirow{4}{*}{0} & A & 0 & 0 & 0 & 0 & 10 \\
\hline & & B & 0 & 0 & 0 & 0 & 10 \\
\hline & & C & 0 & 0 & 0 & 0 & 10 \\
\hline & & $\mathrm{D}$ & 0 & 0 & 0 & 0 & 10 \\
\hline \multirow{4}{*}{ C1 } & \multirow{4}{*}{6,25} & A & 0 & 0 & 1 & 2 & 10 \\
\hline & & B & 0 & 1 & 1 & 2 & 10 \\
\hline & & C & 0 & 0 & 1 & 2 & 10 \\
\hline & & $\mathrm{D}$ & 0 & 1 & 2 & 2 & 10 \\
\hline \multirow{4}{*}{$\mathrm{C} 2$} & \multirow{4}{*}{12,5} & A & 0 & 0 & 0 & 0 & 10 \\
\hline & & B & 0 & 1 & 2 & 3 & 10 \\
\hline & & $C$ & 0 & 1 & 1 & 3 & 10 \\
\hline & & $\mathrm{D}$ & 1 & 1 & 3 & 3 & 10 \\
\hline \multirow{4}{*}{ C3 } & \multirow{4}{*}{25} & A & 0 & 5 & 5 & 5 & 10 \\
\hline & & B & 0 & 2 & 4 & 5 & 10 \\
\hline & & $C$ & 0 & 2 & 3 & 4 & 10 \\
\hline & & $\mathrm{D}$ & 1 & 2 & 4 & 4 & 10 \\
\hline \multirow{4}{*}{ C4 } & \multirow{4}{*}{50} & A & 0 & 5 & 5 & 5 & 10 \\
\hline & & B & 0 & 0 & 1 & 2 & 10 \\
\hline & & $C$ & 2 & 4 & 5 & 6 & 10 \\
\hline & & $D$ & 2 & 4 & 4 & 8 & 10 \\
\hline \multirow{4}{*}{ C5 } & \multirow{4}{*}{100} & A & 0 & 0 & 1 & 3 & 10 \\
\hline & & B & 0 & 1 & 2 & 3 & 10 \\
\hline & & C & 2 & 4 & 6 & 8 & 10 \\
\hline & & $D$ & 2 & 5 & 7 & 10 & 10 \\
\hline
\end{tabular}

Tabla 7. Relación de artefactos navales (chatas)

\begin{tabular}{lll}
\hline \multicolumn{1}{c}{ Puerto } & \multicolumn{1}{c}{ Nombre } & \multicolumn{1}{c}{ Matrícula } \\
\hline Talara & Bahía de Bayóvar & TA-38243-AM \\
& Energy 9501 & TA-38266-AM \\
& Ayelen & CO-21721-AM \\
Santo Domingo & TA-38240-AM \\
Paita & Pelicano & HO-10146-AM \\
& Venus & PT-15236-AM \\
Santa Mónica & PT-16234-AM \\
Salaverry & Copeinca & PT-17458-AM \\
& Pacífico Centro I & CE-21940-AM \\
& PH-2 & PT-15248-AM \\
PH-5 & PT-17532-AM \\
Guanay & CE-10940-AM \\
Inpecasa & CE-01565-AM \\
Chimbote & Chata Jadasa & CE-06619-AM \\
& San Antonio III & CO-17056-AM \\
Tasa Sarimon & CE-01566-AM \\
Tamakun & PT-12174-AM \\
Poseidón & PT-13185-AM \\
San Antonio I & CO-17060-AM \\
Naftes & CE-01590-AM \\
Acuapesca & CE-02413-AM
\end{tabular}




\begin{tabular}{|c|c|c|}
\hline Puerto & Nombre & Matrícula \\
\hline \multirow[t]{5}{*}{ Supe } & Don Oscar & CO-16585-AM \\
\hline & San Antonio II & SE-12182-AM \\
\hline & Petsa 3 & SE-0805-AM \\
\hline & Tasa 2 & SE-0842-AM \\
\hline & Victoria II & SE-00804-AM \\
\hline \multirow[t]{5}{*}{ Huacho } & $\mathrm{PH}-3$ & CE-16699-AM \\
\hline & Chata $N^{0} 2$ & HO-13915-AM \\
\hline & Chata GP-2 & HO-13916-AM \\
\hline & Caracola & HO-10935-AM \\
\hline & América & CE-04042-AM \\
\hline Chancay & Ferjor I & CO-00053-AM \\
\hline \multirow[t]{6}{*}{ Callao } & Alfa & PS-12479-AM \\
\hline & Ventos & CO-13467-AM \\
\hline & Alfa II & PS-18672-AM \\
\hline & Alfa III & PS-12478-AM \\
\hline & Rosita & CO-23465-AM \\
\hline & Bahía III & CO-23415-AM \\
\hline \multirow[t]{5}{*}{ Pisco } & Pisqueña & PS-10857-AM \\
\hline & Exaba & CO-16584-AM \\
\hline & Tangarará & CO-15466-AM \\
\hline & Centinela & PS-13816-AM \\
\hline & Margarita & PS-11981-AM \\
\hline \multirow[t]{8}{*}{ Mollendo } & Gaviota & HO-05473-AM \\
\hline & Alcatraz & MO-3048-AM \\
\hline & Pisco & CO-18469-AM \\
\hline & CFG Doña Teresa & PS-13028-AM \\
\hline & CFG Chancay & CO-12231-AM \\
\hline & Malabrigo & CO-16849-AM \\
\hline & Polar & CO-06241-AM \\
\hline & Vitoria I & MO-06712-AM \\
\hline \multirow[t]{5}{*}{ Ilo } & $\mathrm{PH}-6$ & IO-06716-AM \\
\hline & $\mathrm{PH}-7$ & IO-06053-AM \\
\hline & $D C-3$ & CE-35234-AM \\
\hline & Virgen las Peñas & IO-17236-AM \\
\hline & Chata Ferroles III & PL-05136-AM \\
\hline
\end{tabular}

\section{CONCLUSIONES}

Las pruebas ecotoxicológicas con aguas de lastre y juveniles de Odontesthes regia regia, determinó que éstas representan un impacto significativo en la tasa de mortandad de éstos organismos; además de una notoria diferencia en su calidad ambiental respecto a las aguas de mar de la bahía del puerto del Callao, ya que presenta un $\mathrm{pH}$ muy elevado de 10.48 en comparación al rango establecido de 6.8 - 8.5 en la Categoría 4 de los Estándares de Calidad Ambiental para Agua.

Por otro lado, de los 11 puertos identificados en el Perú respecto a la generación, descarga y disposición de mezclas oleosas se tuvo que durante el año 2017 se gestionó 736.33 m3 de residuos oleosos, para el año 2018 esta demanda creció a $755.65 \mathrm{~m} 3$, mientras que para el año 2019 al mes de mayo se tuvo una cifra de $412.84 \mathrm{~m} 3$.

Asimismo, la generación y disposición de residuos resultante de la gestión de los buques fue de $717.54 \mathrm{t}$ durante el año 2017; $723.21 \mathrm{t}$ en el año $2018 \mathrm{y}$ en el año 2019 hasta el mes de mayo fue de $379.48 \mathrm{t}$.

\section{AGRADECIMIENTOS}

A la Universidad Nacional Mayor de San Marcos, en especial a la Unidad de Posgrado de la Facultad de Ingeniería Geológica, Minera, Metalúrgica y Geográfica y, al Equipo de la Revista del Instituto de investigación. 


\section{REFERENCIAS}

Castillo, G. (Ed). (2004). Ensayos toxicológicos y métodos de evaluación de calidad de aguas. Estandarización, intercalibración, resultados y aplicaciones. México, México: IMTA. Recuperado de https://www.idrc.ca/sites/ default/files/openebooks/147-7/index.html

Deville, D. (2016). Variabilidad morfológica y genética del Pejerrey Odontesthes regia (Humboldt, 1821) en el mar peruano y su implicancia en la determinación de stocks. (Tesis de grado). Universidad Nacional Mayor de San Marcos, Lima, Perú. Recuperado de http://cybertesis. unmsm.edu.pe/handle/cybertesis/8514

Ganoza et al. (2014). Impacto ecológico por extracción ilícita de ovas del Pejerrey (Odontesthes regia regia) su proceso de incubación y eclosión en ambientes controlados. Infinitum 4(2), 121-129. Recuperado de http://revistas.unjfsc.edu.pe/ index.php/INFINITUM/article/view/386

Instituto del Mar del Perú. (2017). Compendio de procedimientos técnicos para la investigación científica en el IMARPE. Callao, Perú. Recuperado de http://biblioimarpe.imarpe. gob.pe:8080/handle/123456789/3177

Madariaga, D. (2010). Modelo de gestión de los residuos procedentes de embarcaciones en los puertos pesqueros y deportivos de Cantabria: Propuestas de control ambiental (Tesis de doctorado). Universidad de Cantabria, Santander, España. Recuperado de https://repositorio.unican.es/xmlui/ handle/10902/1553

Marchant, J. (2009). Investigación sobre la contaminación del mar por basura de naves de crucero en la bahía de Valparaíso años 2002-2009 (Tesis de maestría). Universidad de Concepción, Flacso, Chile. Recuperado de https:// repositorio.flacsoandes.edu.ec/handle/10469/6573

Organización Marítima Internacional. (1973). Convenio Internacional para prevenir la Contaminación por los Buques - MARPOL 73/78. Recuperado de http://www.imo. org/es/About/Conventions/ListOfConventions/Paginas/ International-Convention-for-the-Prevention-of-Pollutionfrom-Ships-(MARPOL).aspx
Organización Marítima Internacional. (2005). Convenio sobre la gestión del agua de lastre. London, Reino Unido: IMO. Recuperado de http://www.imo.org/es/OurWork/ Environment/BallastWaterManagement/Documents/ BWM2Circ13Rev1es.pdf

Orosco, P. y Villanueva, H. (2015). Toxicidad aguda en alevines de Pejerrey (Odontesthes regia, Humboldt 1821) empleando aguas residuales del colector "La Chira", Chorrillos-Lima (Tesis de pregrado). Universidad Nacional del Callao, Callao, Perú. Recuperado de http://repositorio.unac.edu.pe/ handle/UNAC/1555

Ramírez, F. (2015). El régimen internacional de la bioinvasión marina causada por agua de lastre: Especial referencia a la República de Colombia (Tesis de doctorado). Universidad Autónoma de Barcelona, Barcelona, España. ISBN: 9788449053293. Recuperado de https:/www.tdx.cat/ handle $/ 10803 / 311418 \#$ page $=1$

Salcedo, C. (2016). Diseño de un sistema de recepción, transporte $y$ tratamiento primario de mezclas oleosas en un terminal portuario (Tesis de maestría). Universidad de Guayaquil, Guayaquil, Ecuador. . Recuperado de http://repositorio. ug.edu.ec/handle/redug/35541

Sánchez, G. y Vera, G. (2001). Manual Introductorio de Ecotoxicología Acuática del Instituto del Mar del Perú. Callao, Perú: IMARPE. Recuperado de https://core.ac.uk/ download/pdf/47267075.pdf

Torres, G. (2015). Evaluación de mareas rojas durante 19682009 en Ecuador. Acta Oceanográfica del Pacifico, (20)1, p. 89-98. Recuperado de https://www.inocar.mil.ec/ web/phocadownloadpap/actas_oceanograficas/acta20/ OCE20_7.pdf

Vera et al. (22 de julio del 2001). Pruebas ecotoxicológicas con cadmio y cromo usando postlarvas del pejerrey Odontesthes (Austromenidia) regia regia Hildebrand. Revista Peruana de Biología. Volúmen (8). p. 125-135. DOI https://doi. org/10.15381/rpb.v8i2.6715 
